\title{
Jet Measurements In CMS
}

\section{Sanmay Ganguly*}

Department Of High Energy Physics

Tata Institute Of Fundamental Research

Mumbai, India

E-mail: Sanmay.Ganguly@cern.ch

\begin{abstract}
A measurement of inclusive jet and dijet production cross sections is presented [2]. Data from large hadron collider (LHC) proton-proton collisions at $\sqrt{s}=7 \mathrm{TeV}$, corresponding to $4.67 \mathrm{fb}^{-1}$ of integrated luminosity, have been collected with the compact muon solenoid (CMS) detector [1]. Jets are reconstructed with the anti- $k_{T}$ clustering algorithm with size parameter $R=0.7$, extending to rapidity $|y|=2.5$, transverse momentum $p_{T}=2 \mathrm{TeV}$, and dijet invariant mass $M_{J J}=5 \mathrm{TeV}$. The measured cross sections are corrected for detector effects and compared to perturbative QCD predictions at next-to-leading order (NLO), corrected for non perturbative (NP) factors, using various sets of parton distribution functions.

Determination Of Jet Energy Correction from $\sqrt{s}=7 \mathrm{TeV}$ CMS data is presented. The individual components are determined. The jet energy scale uncertainty factors are also shown.
\end{abstract}

36th International Conference on High Energy Physics,

July 4-11, 2012

Melbourne, Australia

${ }^{*}$ Speaker. 


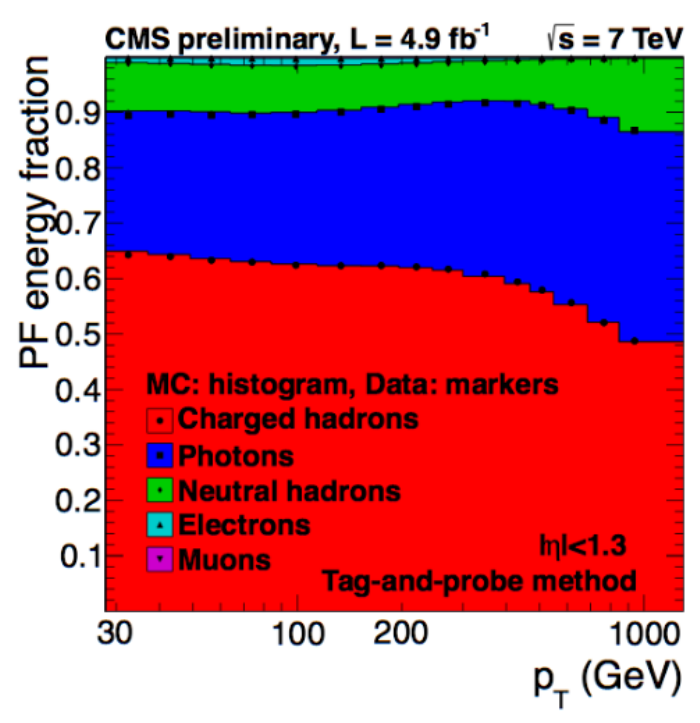

Figure 1: Jet compositions

\section{Introduction}

At LHC proton-proton collision is taking place at very high center of mass energy $\sqrt{s}=7$ $\mathrm{TeV}$. At such energy scale, the fundamental interacting objets are partons, e.g. quarks, gluons etc. After the collision, the hard scattered partons undergoes fragmentation and then hadronization to form colorless bound states. These colorless objects deposit energy at dfferent components of the detector. To study the parton level dynamics, jet clustering algorithm is applied on each stable particles in an event, reconstructed from every individual component of the detector.

Jet study is important from both thoretical and experimental point of view. It is also used to improve different parton shower model and higher order theory prediction. Measured cross section of jet processes are often used to estimate the number of background events for new physics studies, as well as constrain the parameters of parton distribution function.

\section{Jet Reconstruction}

In this presentation we have discussed about jet, which are reconstructed by particle flow (PF) algorithm. PF algorithm is an event reconstruction technique which attempts to reconstruct and identify all stable particles in an event. It combines all information from all the sub detectors viz. tracker, electromagnetic calorimeter (ECAL), hadronic calorimeter (HCAL), muon chamber. The stable particles reconstructed from these subdetectors are $\mu^{+}, \mu^{-}, e^{+}, e^{-}, \gamma, \pi^{+}, \pi^{-}, \pi^{0}, K^{+}$, $K^{-}, K^{0}$ etc. Jet reconstruction algorithm is applied on this final state stable particles to reconstruct jets. Figure 1 shows the data montecarlo comparison of jet energy fraction of the constituents. In our current presentation, we have shown analysis with jets reconstructed by anti-kT algorithm, using size parameter $\mathrm{R}=0.7$ ( $\mathrm{ak} 7$ ). The clustering is done using FastJet package. 


\section{Jet Energy Correction}

Jets are reconstructed from stable particles in the detector. So jet energy and momenta are effected by detector level noise, high pileup, varying detector response etc. The purpose of jet energy correcion (JEC) is to relate, on average, the enrgy measured for the detector jet corresponding to the particle level jet.[5] A true particle level jet results from clustering of all stable particles originating from fragmentation of hard scattered partons, as well as particles orginating from underlying event activity.

In CMS, the JEC is applied on each momentum component of the reconstructed jet as a multiplicative factor as $p_{\mu}^{\text {cor }}=\mathscr{C} \cdot p_{\mu}^{\text {raw }}$, where the correction factor $\mathscr{C}$ is product of several components as,

$$
\mathscr{C}=C_{1}(\text { Offset }) \times C_{2}(\text { Relative }) \times C_{3}(\text { Absolute }) \times C_{4}(\text { Residual })
$$

Here $C_{1}($ Offset $)$ is the correction factor which removes the contribution coming from pile-up. $C_{2}$ (Relative) corrects for the non linear $\eta$ (pseudo rapidity $\eta=-\ln \left(\tan \frac{\theta}{2}\right)$ ) dependence of the detector response. $C_{3}$ (Absolute) for the non linear jet transverse momentum $\left(p_{T}\right)$ dependence of the detector response and $C_{4}$ (Residual) is the residual correction factor which accounts for the mismatch between data and montecarlo prediction.

The $C_{1}($ Offset $)$ factor is measured by average offset method and jet area method.

$C_{2}$ (Relative) factor method is determined by dijet balance method and $C_{3}$ (Absolute) is factor is determined by $\gamma / Z+$ jet $p_{T}$ balance method. Figure 2 shows the individual JEC factors and uncertainty due to several factors which are uncorrelated among themselves.

\section{Inclusive And Dijet Measurements}

In this talk we have mainly discussed about the measurement of double differential cross section of inclusive jet $(p+p \rightarrow j e t+X)$ and dijet $(p+p \rightarrow j e t+j e t+X)$ production as a function of inclusive jet $p_{T}$ and dijet invariant mass $\left(M_{j j}\right)$ for different rapidity bins from $\sqrt{s}=7 \mathrm{TeV} \mathrm{CMS}$ data[2]. The measured cross sections are corrected for detector effects and compared to the QCD predictions. The parton transverse momentum fractions $x_{T}=\frac{2 p_{T}}{\sqrt{s}}$ probed in this measurement cover the range $0.033<x_{T}<0.57$.

The jets are selected with tight jet identification criteria, to reject detector level noise, followed by JEC applied on selected jets. The double differential cross section are measured using the formula

$$
\begin{aligned}
& \frac{d^{2} \sigma}{d p_{T} d|y|}=\frac{1}{\varepsilon L} \frac{N}{\Delta p_{T} \Delta|y|} \times C_{\text {unsmearing }} \\
& \frac{d^{2} \sigma}{d M_{j j} d|y|}=\frac{1}{\varepsilon L} \frac{N}{\Delta M_{j j} \Delta|y|} \times C_{\text {unsmearing }}
\end{aligned}
$$

where $L$ is the total integrated luminosity, $\varepsilon$ is the trigger efficiency, $N$ is the number of jets in a bin, where $\Delta p_{T}, \Delta M_{j j}$ are the bin width and $C_{\text {unsmearing }}$ is the non perturbative correction factor.

For the inclusive jet measurement, events are required to contain at least one tight jet with $p_{T}>114$ $\mathrm{GeV}, 196 \mathrm{GeV}, 300 \mathrm{GeV}, 362 \mathrm{GeV}$, and $507 \mathrm{GeV}$ for the five single-jet HLT triggers used respectively. For the dijet measurement, at least two tight reconstructed jets with $p_{T 1}>60 \mathrm{GeV}$ and $p_{T 2}>$ $30 \mathrm{GeV}$ are required. 

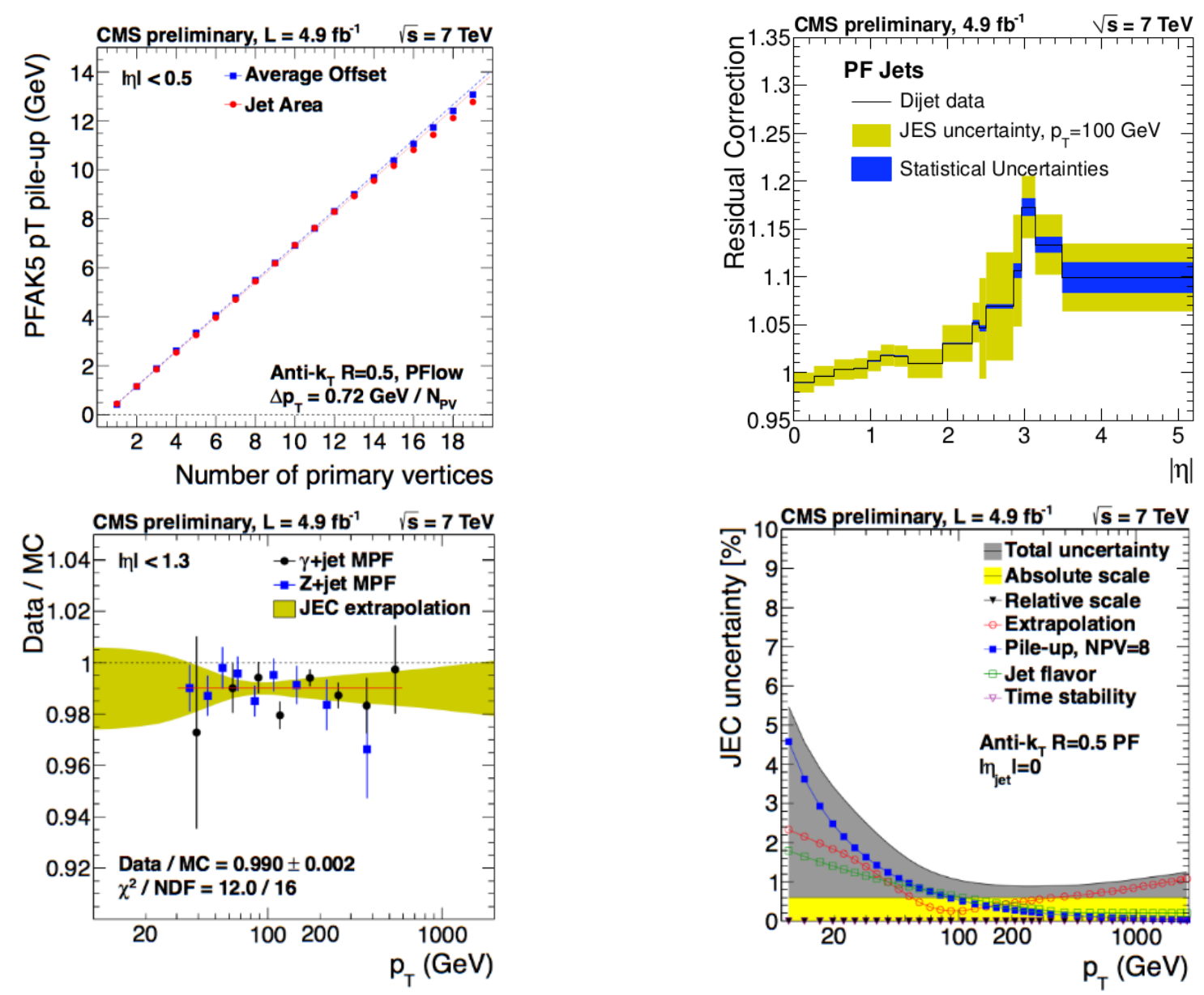

Figure 2: JEC factors and JES Uncertainties.
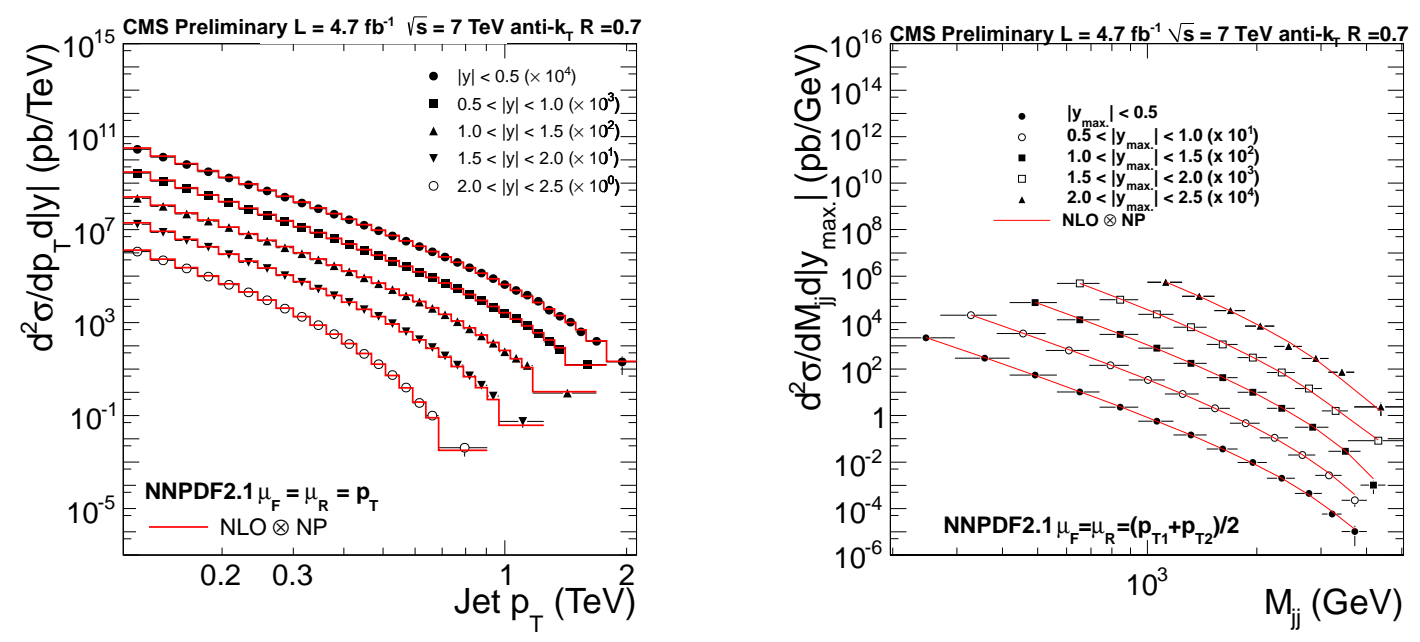

Figure 3: Inclusive and Dijet double differential cross section compared with $N L O \times N P$ theory prediction 

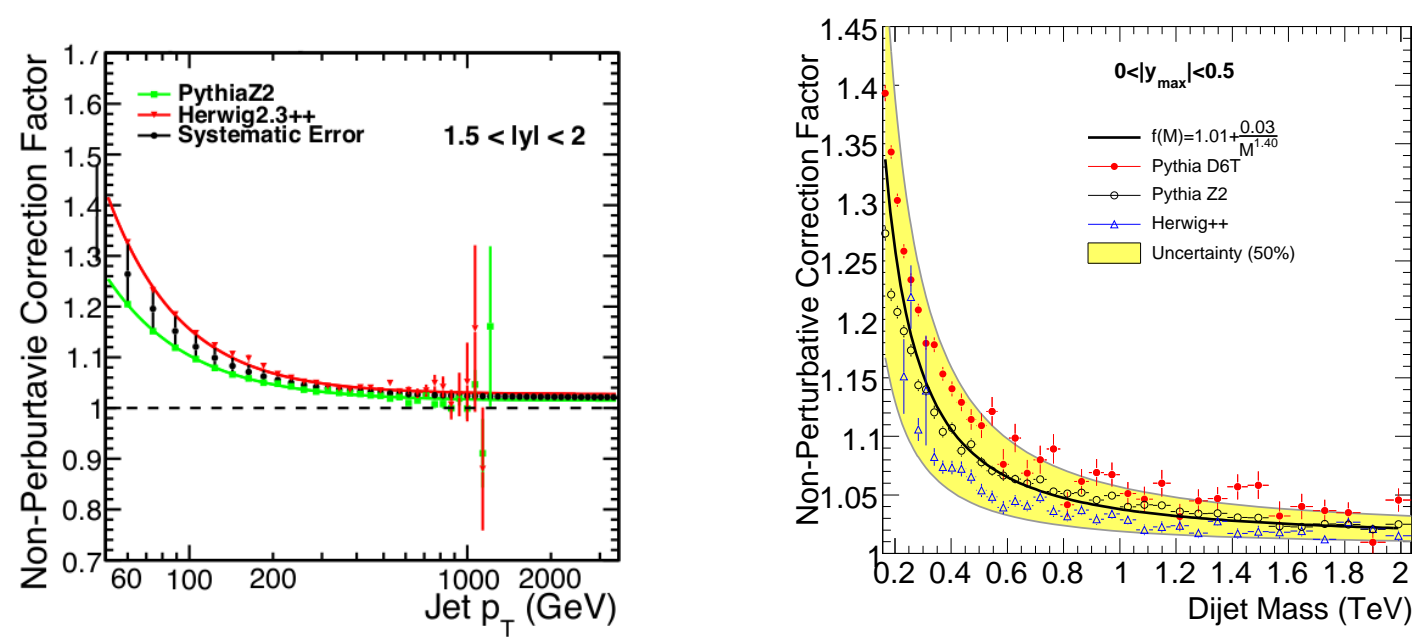

Figure 4: NP correction factor for inclusive jet and dijet

Figure 3 shows the double differential spectrum for different rapidity bins extending upto $|y|=2.5$. The measured spectra is then unfolded using Bayesian unfolding (D'Agostini)[4] technique to get rid of the detector resolution effects and then compare directly to the particle level spectrum. Comparison with $N L O \times N P$ theory has been done for several parton distribution function (PDF) sets.

The non perturbative correction factor is applied on next to leading order (NLO) spectrum to account for hadronization effects and multi parton interactions (MPI). This factor is computed as the ratio of the observables, with once switching on and then switching off the hadronization and MPI effects. In the current analysis the NP currection factor is evaluated from two generators, Pythia6 and Herwig++. The difference in the factor derived from these two event generators is counted as the uncertainty introduced due to NP correction on the measured cross section. Figure 4 shows the variation of NP correction factor as a function of jet $p_{T}$ and $M_{j j}$.

The NLO theory calculation is done by NLOJet++ package using fastNLO program[6], the renormalization and factorization scale has been set equal to inclusive jet $p_{T}$ and $M_{j j}$ in the individual cases. The scale uncertainty is evaluated by varying the scales for six points as $\frac{\mu_{F}}{\mu}, \frac{\mu_{R}}{\mu}=$ $(2,2),(0.5,0.5),(1,0.5),(0.5,1),(2,1),(1,2)$. The total theoretical uncertainty varies upto $30 \%$, where as the experimental uncertainty varies between $10 \%$ to $30 \%$. The NLO theoretical calculations are done for five different PDF sets viz. ABKM09, MSTW2008, HERA15, CT10, NNPDF2.1 . Figure 5 shows data over theory plots for both the variables in central and outer most rapidity bin. The figure shows that the measured spectrum is in good agreement with the NLO theory spectrum within experiemntal and theoretical uncertainty limits.

\section{Conclusion}

In this talk first we have discussed about the jet reconstruction and jet energy corrections and finally inclusive and dijet measurements from $7 \mathrm{TeV}$ CMS data. A discussion on jet reconstruction from particle flow algorithm is done. Determination of individual JEC factors from data are 

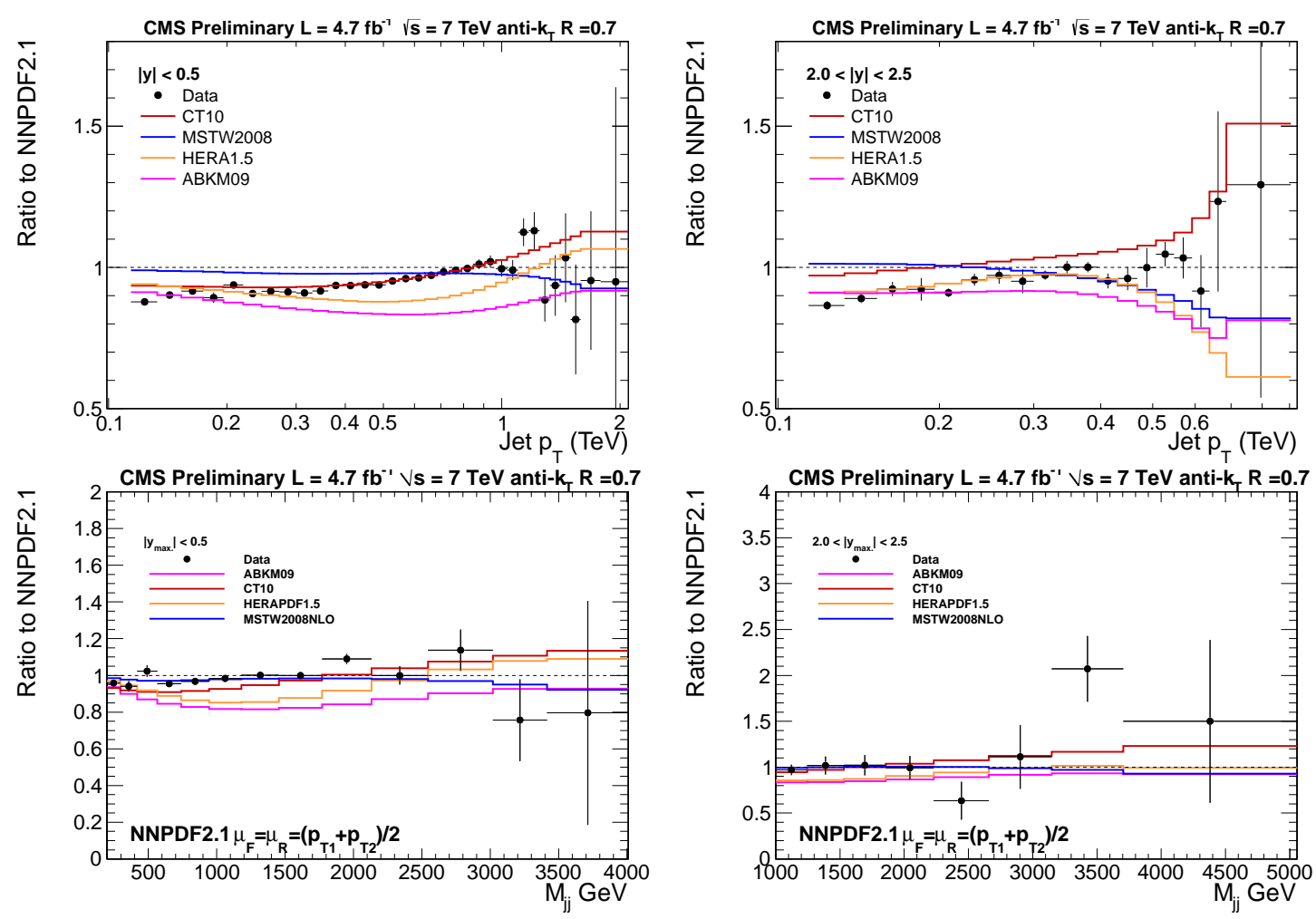

Figure 5: Data over theory ratio with total uncertainty limits.

discussed. Then we proceed to discuss about the double differential cross section measurement for inclusive and dijet production cross section from p-p collision. A comparison of unfolded measured cross section with $N L O \times N P$ theory spectrum is presented for five different PDF state for each rapidity bins.

Estimation of theoretical and experimental uncertainties have been discussed and it is shown that the data over theory ratio is well within the uncertainty bands.

This measurement can be used for PDF fittings and strong coupling constant extraction.

\section{References}

[1] The CMS experiment at the CERN LHC

The CMS Collaboration, 2008 JINST 3 S08004

[2] Jet Cross Section and PDF Constraints CMS-PAS QCD-11-004

[3] Measurement of inclusive jet and dijet production in pp collisions at 7TeV using the ATLAS detector. ATLAS Collaboration, Phys.Rev.D 86,014022 (2012)

[4] Unfolding algorithms and tests using RooUnfold.

T. Adye, Arxiv:data-an/1105.1160

[5] Determination of Jet Energy Calibration and Transverse Momentum Resolution in CMS CMS Collaboration, JINST, Vol. 6, 2011

[6] fastNLO: Fast pQCD calculations for PDF fits T. Kluge, K. Rabbertz, M. Wobisch, Arxiv:hep-ph/0609285v2 